\title{
ON THE ENERGY SPECTRUM FOR WEAK SOLUTIONS OF THE NAVIER-STOKES EQUATIONS
}

\author{
ANNA L. MAZZUCATO
}

\begin{abstract}
We consider the decay at high wavenumbers of the energy spectrum for weak solutions to the three-dimensional forced Navier-Stokes equation in the whole space. We observe that known regularity criteria imply that solutions are regular if the energy density decays at a sufficiently fast rate. This result applies also to a class of solutions with infinite global energy by localizing the Navier-Stokes equation. We consider certain modified Leray backward self-similar solutions, which belong to this class, and show that their energy spectrum decays at the critical rate for regularity. Therefore, this rate of decay is consistent with the appearance of an isolated self-similar singularity.
\end{abstract}

\section{INTRODUCTION}

In this note, we investigate the decay rate of the energy spectrum for weak solutions to the three-dimensional forced Navier-Stokes equation (NS for short):

$$
\begin{aligned}
& \partial_{t} u(x, t)+u \cdot \nabla u(x, t)-\nu \Delta u(x, t)+\nabla p(x, t)=f(x, t), \\
& \operatorname{div} u(x, t)=0,
\end{aligned}
$$

where $u$ is the velocity vector field, $p$ is the pressure scalar field, $f$ are body forces, and $\nu$ is the viscosity coefficient.

Informally, the energy spectrum $E(\kappa, t)$ is defined as the average energy density at a certain length scale $\ell$. This scale corresponds to a wavenumber amplitude $\kappa \sim 1 / \ell$ in frequency space. The behavior of $E(\kappa, t)$ is central to turbulence theory. In particular, it is proportional to the rate of energy dissipation per wavenumber amplitude, which determines the local energy cascade. It is well-known that if a solution of (1.1) is regular enough, then the energy spectrum is exponentially decaying [DT95]. Our approach is in some sense opposite, that is, we are concerned with the minimal rate of decay that will ensure regularity. Furthermore, we do not require a control point-wise in time on $E(\kappa, t)$. We therefore average $E(\kappa, t)$ over some finite interval $[0, T]$ and we recast the decay of the time-averaged spectrum $\bar{E}(\kappa)$ in terms of regularity in Sobolev spaces.

We investigate the sharpness of this condition by computing the energy spectrum for certain modified Leray backward self-similar solutions of the unforced NavierStokes equation, which develop a singularity at an isolated point in space and time. Such singularities are the simplest, although it is not known whether all isolated

Date: July 1, 2004.

2000 Mathematics Subject Classification. 35Q30, 76D03, 76F05, 42B35, 42C40.

The author would like to thank Vladimir Sverak for his help and support, her Ph.D. adviser Michael Taylor for his continuous interest, and the anonymous referees for their insightful comments. This work was partially conducted while the author was a Post-doctoral Fellow at the Institute for Mathematics and its Applications, and a Gibbs Instructor at Yale University. 
singularities must be self-similar. Moreover, parabolic rescaling in space-time gives the asymptotic behavior of a solution near a (possible) singularity and the limit of these "blow-ups" is a self-similar solution.

The backward Leray solutions that we study can formally be obtained by dynamic rescaling and their interest is motivated by comparison with the complex Ginzburg-Landau equation [PŠ01]. Their existence cannot be excluded based on generalized energy inequalities as for the standard backward self-similar solutions [Tsa98],[NRŠ96]. We impose the slowest decay at infinity that is consistent with local energy inequalities and partial regularity in the sense of Caffarelli-KohnNirenberg [CKN82] (after previous work of Scheffer [Sch76] and Foias-Temam [FT79] ), that is, homogeneous of degree -1 . In particular, these self-similar solutions are suitable weak solutions with globally infinite energy.

Consequently, while we employ known regularity criteria for weak solutions, especially the recent results in [KT00], [BdV95], we apply them to a class of weak solutions with (possibly) global infinite energy by means of a certain "localization" of the equation. The localization procedure introduces several technical difficulties, such as establishing a priori bounds on the pressure. Additionally, localizing NS creates a fictitious force $f$ in the equation, depending on $u$ and $p$. Controlling the regularity of $f$ is another delicate issue. By a weak solution to NS, we therefore mean a vector field $u$ on $\mathbb{R}^{3} \times[0, T]$ that satisfies (1.1) in the sense of distributions for some scalar distribution $p(x, t)$ on cylinders $D \times[0, T]$, where $D$ is a bounded domain in $\mathbb{R}^{3}$. $u$ is required to have finite norm only in $L^{\infty}\left([0, T], L^{2}(D)\right)$.

Solutions of this form, satisfying some additional conditions, were recently constructed by P. G. Lemarié [LR99]. Initial data that do not belong to $L^{2}\left(\mathbb{R}^{3}\right)$ appear naturally as well when solving (1.1) by semigroup methods with an approach pioneered by T. Kato [Kat83] and F. Weissler [Wei80]. The correct behavior of the solution under dilations is crucial in this context and therefore initial data have typically higher local regularity but slower decay at infinity than $L^{2}$ functions. We investigated mild solutions with initial data in some modified Besov spaces in [Maz03].

Scaling considerations suggest that the minimal decay rate of $\bar{E}(\kappa)$ ensuring regularity is a rate that is $o\left(\kappa^{-4}\right)$, which is equivalent to saying that the solution $u$, after localization, belongs to $L^{2}\left([0, T], H^{3 / 2}\right)$. We show that the modified backward self-similar solutions described above have an energy spectrum satisfying precisely the expected critical decay rate, that is,

$$
\bar{E}(\kappa)=O\left(\kappa^{-4}\right)
$$

but otherwise meet all the requirements to apply the regularity criteria. In particular, we can obtain a priori estimates on the pressure and forcing due to localization by rescaling in space and time, exploiting the homogeneity of the solution at infinity.

As mentioned, Doering and Titi [DT95], following Foias and Temam [FT89], proved that the energy spectrum is exponentially decaying in $\kappa$, after an initial transient time, if $\|\nabla u(\cdot, t)\|_{L^{2}}$ is uniformly bounded in time.

Decay rates in terms of Gevrey-class regularity are treated in depth by Foias, Manley, Rosa, Temam in [FMRT01]. Frisch and Sulem [SF75] showed that a power law with exponent $-8 / 3$ is the maximum rate of decay for the spectrum that can sustain an energy cascade when the total energy is finite. Doering and Gibbon [DG02] recently studied moments of the energy spectrum. In particular, they concluded that exponents $q<-8 / 3$ are not consistent with a finite energy dissipation 
rate $\epsilon$ in the zero-viscosity limit. Our bounds are clearly far from this range and, moreover, we consider high frequencies, possibly well beyond the dissipation scale. These estimates, though perhaps not informative from the point of view of turbulence, provide nevertheless information on the behavior of weak solutions and are rigorously obtained from the equation.

The paper is organized as follows. In Section 2, we give a precise notion of weak solutions on space-time cylinders and define the energy spectrum for these solutions. In Section 3, we consider finite-energy weak solutions $u$ to the Navier-Stokes equation with forcing term $f$. We prove in Theorem 1 that, if $f \in L^{r}\left([0, T], L^{2}\left(\mathbb{R}^{3}\right)\right)$, $r>2$, and $u \in L^{2}\left([0, T], H^{3 / 2}\left(\mathbb{R}^{3}\right)\right)$, then $u$ coincides with a strong solution to NS in the class $C\left([0, T], H^{1}\left(\mathbb{R}^{3}\right)\right.$. Finally, in Section 4 , we introduce modified Leray backward self-similar solutions of the form

$$
u(x, t)=\frac{1}{\sqrt{T-t}} U\left(\frac{x}{\sqrt{T-t}}, \tau\right)
$$

where $U$ is a smooth, divergence-free vector field, periodic in $\tau$, and $\tau$ is related to $t$ by $\tau=\log \frac{T}{T-t}$. $T$ is therefore the blow-up time, but we assume that $u$ continues as a weak solution on $\left(T, T^{\prime}\right), T^{\prime}>T$. The singular profile at blow-up is precisely a -1 -homogeneous function. We then localize the Navier-Stokes equation to a large ball $B(0, R)$ via a smooth cut-off, and prove that the forcing term introduced by the localization satisfies the condition of Theorem 1. We show in Proposition 3, using a wavelet decomposition, that the $L^{2}\left([0, T], H^{3 / 2}\left(\mathbb{R}^{3}\right)\right)$ norm is barely divergent. In fact, $u \in L^{2, \infty}\left([0, T], H^{3 / 2}\left(\mathbb{R}^{3}\right)\right)$. It seems difficult to use the Fourier transform directly to compute the Sobolev norm, given that $u$ is homogeneous only at time $t=T$ and we apply a cut-off function $\eta$. Finally, in Proposition 4 we estimate the decay of the Littlewood-Paley spectrum of $u$, introduced by Constantin [Con97], which lends itself to a complete characterization in terms of wavelet coefficients.

We state all our results in the three-dimensional case, but analogous conclusions can be drawn in any dimension.

We close this introduction by setting some notation. Given a distribution $f$, we denote by $\hat{f}$ and $\check{f}$ respectively its Fourier and Inverse Fourier transforms. We use the symbol $\asymp$ to indicate equivalence of norms, while $\approx$ represents the usual asymptotic relation (see e.g. [BO99]). For $f$ and $g \in L^{2}\left(\mathbb{R}^{3}\right),(f, g)$ stands for the usual $L^{2}$-inner product. Finally, we write $C$ for any non-essential constant.

\section{Definitions}

Let $D$ be an open subset of $\mathbb{R}^{3}$ and $T>0$. We denote the subspace of $L^{2}\left(\mathbb{R}^{3}\right)$ of divergence-free fields by $L_{\sigma}^{2}\left(\mathbb{R}^{3}\right)$, and similarly for the $\left(L^{2}\right.$-based) Sobolev spaces $H^{s}\left(\mathbb{R}^{3}\right)$.

Definition 2.1. A weak solution $u$ to (1.1) on $D \times[0, T]$ is a measurable function $u$ on $\mathbb{R}^{3} \times[0, T]$ such that:

(i) $u \in L^{\infty}\left([0, T], L_{\sigma}^{2}(D)\right) \cap L^{2}\left([0, T], H_{\sigma}^{1}(D)\right)$,

(ii) $u$ is continuous in time in the weak topology of $L_{\sigma}^{2}(D)$, 
(iii) $\forall \phi \in C_{0}^{\infty}(D \times[0, T]), \operatorname{div} \phi=0$,

$$
\begin{aligned}
& \int_{0}^{T} \int_{D} u(x, s) \cdot \partial_{s} \phi(x, s) d x d s-\int_{0}^{T} \int_{D} \nabla u(x, s) \cdot \nabla \phi(x, s) d x d s+ \\
&+\int_{0}^{T} \int_{D}(u \cdot \nabla u)(x, s) \cdot \phi(x, s) d x d s+\int_{0}^{T} \int_{D} f(x, s) \cdot \phi(x, s) d x d s= \\
& \quad=\int_{D} u(x, T) \cdot \phi(x, T) d x-\int_{D} u_{0} \cdot \phi(x, 0) d x
\end{aligned}
$$

for some $u_{0} \in L_{\sigma}^{2}(D)$.

In what follows, we will omit an explicit reference to the underlying space $D$, whenever it is clear which domain we are considering.

There is no information on the pressure in the weak formulation. However, the regularity of $u$ implies the existence of a function $p \in L^{5 / 3}\left([0, T], L^{5 / 3}(D)\right)$ such that NS is satisfied in the sense of distributions (see [Lin98] and also [SŠ01] for a discussion on this point).

Let now $u$ be a weak solution to NS on $D=\mathbb{R}^{3}$.

Definition 2.2. The energy spectrum of $u$ is defined as the unique integrable function $E(\kappa, t)$ on $\mathbb{R}^{+}$given by:

$$
\int_{0}^{\infty} E(\kappa, t) d \kappa=\int_{\mathbb{R}^{3}}|\hat{u}(k, t)|^{2} d k .
$$

By Fubini's theorem, for a. e. $\kappa$

$$
E(\kappa, t)=\int_{|k|=\kappa}|\hat{u}(k, t)|^{2} d k .
$$

The energy spectrum is often called the power spectrum of $u$. $E(\kappa, t)$ is a relevant quantity in isotropic turbulence, where it is expected that the local average energy at scales of order $2 \pi / \kappa$ depends only on the wavenumber amplitude $\kappa$ and the bulk rate of energy dissipation $\epsilon$ (see e.g. [DG95], [Fri95], [FMRT01]).

Definition 2.3. We define the time-averaged energy density $\bar{E}$ by:

$$
\int_{0}^{\infty} \bar{E}(\kappa) d \kappa=\frac{1}{T} \int_{\mathbb{R}^{n} \times[0, T]}|\hat{u}(k, t)|^{2} d k .
$$

If $u$ is only locally in $L^{2}$, we associate to any open, bounded set $D \subset \mathbb{R}^{3}$ a "local" power spectrum $\bar{E}_{D}(\kappa)$, defined as the power spectrum of $\eta u$, where $\eta \in C_{0}^{\infty}\left(\mathbb{R}^{3}\right)$ is a smooth cut-off function such that $\eta \equiv 1$ on $D$.

In this paper we consider the decay rate in $\kappa$ of the energy spectrum that is naturally associated to the scaling of the Navier-Stokes equation. We show that this rate is sharp for certain (hypothetical) Leray self-similar solutions that develop a singularity at an isolated space-time point. We impose the weakest behavior at infinity consistent with partial regularity [Ler33],[CKN82], i.e.,

$$
u(x, t)=O\left(\frac{1}{|x|}\right) \text {, as } x \rightarrow \infty .
$$

In particular, these solutions do not have a globally-finite energy norm. 
We recast the decay of $\bar{E}(\kappa)$ as finiteness of certain Sobolev norms weighted in time. It is convenient in this context to work with the so-called LittlewoodPaley energy spectrum $E_{L P}(k, t)$, introduced by Constantin [Con97], and its timeaveraged version $\bar{E}_{L P}(k, t)$.

Let $\tilde{\phi}_{0}(k), \tilde{\psi}_{j}(k), j \in \mathbb{Z}$, be the components of a Littlewood-Paley partition of unity, satisfying:

$$
\sum_{j \geq 0} \tilde{\psi}_{j}+\tilde{\phi}_{0}=1
$$

with convergence in the sense of distributions. Each $\tilde{\psi}_{j}$ is a bump function supported on the dyadic shell $D_{j}=\left\{k\left|2^{j-1}<\right| k \mid<2^{j+1}\right\}$, which is constructed by dilating a single function $\tilde{\psi}$ :

$$
\tilde{\psi}_{j}(k)=\tilde{\psi}\left(2^{-j} k\right),
$$

while $\tilde{\phi}_{0}$ is a bump function on the ball $B(0,2)$. Then, for $k \in\left(2^{j-1}, 2^{j+1}\right), j \in \mathbb{Z}$, we set:

$$
E_{L P}(k, t)=|k|^{-1}\left\|\tilde{\psi}\left(2^{-j} \cdot\right) \hat{u}(\cdot, t)\right\|_{L^{2}}^{2} .
$$

In the next section we derive a regularity criterion for weak solutions to the Navier-Stokes equation that is applicable to solutions having decay (2.3) under some additional assumptions. These assumptions are satisfied by the modified Leray backward self-similar solutions. The main tool consists in "localizing" the equation to a large ball. This localization procedure introduces a fictitious forcing that needs to be controlled.

\section{Decay Rates}

We consider weak solutions to the Navier-Stokes equation on $\mathbb{R}^{3} \times[0, T]$ in the sense of definition 2.1 with forcing term $f$.

Theorem 1. Let $u$ be a weak solution to the Navier-Stokes equation with forcing $f \in L^{r}\left([0, T], L^{2}\left(\mathbb{R}^{3}\right)\right)$, for some $r>2$. Assume further that:

$$
\nu^{-1} \int_{0}^{\infty} \kappa^{3} \bar{E}(\kappa) d \kappa<\infty .
$$

Then, $u$ is regular on $(0, T]$.

By $u$ regular, we mean $u \in H^{1}\left(\mathbb{R}^{3}\right)$, continuously in time. In fact, in this case, if $f$ is smoother, one can bootstrap the regularity of $u$.

Since $u \in L^{2}\left([0, T], H^{1}\left(\mathbb{R}^{3}\right)\right)$, condition (3.1) is equivalent to

$$
\nu^{-1}\|u\|_{L^{2}\left([0, T], H^{3 / 2}\right)}<\infty .
$$

Furthermore, in (3.1) we can use the Littlewood-Paley spectrum $\bar{E}_{L P}(k, t)$ instead, given that:

$$
\begin{aligned}
\int_{0}^{\infty} \kappa^{3} \bar{E}(\kappa, t) d \kappa & \asymp \int_{0}^{\infty} \kappa^{3}\left(\frac{1}{T} \int_{0}^{T}\left\|\tilde{\psi}\left(\kappa^{-1} \cdot\right) \hat{u}(\cdot, t)\right\|_{L^{2}}^{2} d t\right) \frac{d \kappa}{\kappa} \\
& \asymp \int_{0}^{\infty} \kappa^{3} \bar{E}_{L P}(\kappa, t) d \kappa .
\end{aligned}
$$

When $f \equiv 0$, Theorem 1 is an immediate consequence of the following regularity criteria recently obtained by Kozono and Taniuchi [KT00], and Beirão da Veiga [BdV95]. Specifically, Kozono and Taniuchi proved that a weak solution $u$ of the 
Navier-Stokes equation on $\mathbb{R}^{3} \times[0, T]$ agrees for every $0<\epsilon<T$ with a strong solution in the class $C\left([\epsilon, T), H^{s}\right) \cap C^{1}\left((\epsilon, T), H^{s}\right) \cap C\left((\epsilon, T), H^{s+2}\right), \forall s>1 / 2$, if

$$
\int_{0}^{T}\|u(\cdot, t)\|_{\mathrm{BMO}}^{2}<+\infty
$$

where BMO is the space of functions of bounded mean oscillation of John and Nirenberg [JN61]. This result improves on the limiting case, $p=\infty, q=2$, of Serrin's condition for regularity [Ser62], [Str88], [Gig86]:

$$
u \in L^{q}\left((0, T), L^{p}\right), \quad \frac{2}{q}+\frac{3}{p} \leq 1, \quad p>3 .
$$

For Leray-Hopf solutions, (K-T) is also sufficient for uniqueness [KT00]. Beirão da Veiga obtains a similar conclusion when

$$
\int_{0}^{T}\|\nabla u(\cdot, t)\|_{L^{r}}^{\alpha}<+\infty, \quad \frac{2}{\alpha}+\frac{3}{r}=2,
$$

for $1<\alpha<\infty, 3 / 2<r<\infty$.

In three dimensions, $H^{3 / 2}$ corresponds to the critical case for the Sobolev inequality.

While $H^{3 / 2}$ contains unbounded functions, oscillations at different scales are controlled. In fact, $H^{3 / 2}$ is a subset of BMO (see e.g. [Tri92]). This embedding follows from the Littlewood-Paley characterization of BMO and $H^{3 / 2}$ :

$$
\begin{aligned}
& \|f\|_{\mathrm{BMO}} \asymp \sup _{\mathrm{J} \text { dyadic }}\left(\frac{1}{|\mathrm{~J}|} \int_{\mathrm{J}} \sum_{j \geq-\log _{2} \ell(\mathrm{J})}\left|\tilde{\tilde{\psi}}_{j} * f\right|^{2}\right)^{1 / 2}, \\
& \|f\|_{H^{3 / 2}} \asymp\left\|\tilde{\tilde{\phi}}_{0} * f\right\|_{L^{2}}+\left(\sum_{j \geq 0} 2^{3 j}\left\|\tilde{\tilde{\psi}}_{j} * f\right\|_{L^{2}}^{2}\right)^{1 / 2},
\end{aligned}
$$

where J are dyadic cubes, and Berstein's inequality:

$$
\left\|\tilde{\tilde{\psi}}_{j} * f\right\|_{L^{\infty}} \leq 2^{3 j / 2}\left\|\tilde{\tilde{\psi}}_{j} * f\right\|_{L^{2}} .
$$

At the same time, $H^{3 / 2} \subset W^{1,3}$, and regularity is also obtained from (BdV) with $\alpha=2, r=3$.

The methods used by Kozono and Taniuchi can be adapted to include a nontrivial forcing term.

We begin with a technical lemma. We will need to bound some non-linear quantities in terms of Sobolev norms. These estimates are known (cf. Lemma 1 in [KT00]), but for completeness we indicate a proof. Below, if $\phi$ is a smooth function, $\phi(D)$ represents the usual associated Fourier multiplier, given by the formula:

$$
(\widehat{\phi(D) f})(k)=\phi(k) \hat{f}(k) .
$$

Lemma 2. Let $\alpha, \beta=1, \ldots, 3$ and $u \in L^{2}$ a given function. Then,

$$
\left\|\partial_{\alpha} u \partial_{\beta} u\right\|_{L^{2}} \leq \bar{C}\|u\|_{H^{3 / 2}}\|\Delta u\|_{L^{2}},
$$

where $\bar{C}$ is a positive constant, independent of $u$. 
Proof. Estimate (3.6) is established by means of a high-low frequency decomposition of a product (we refer to [Tay91] for a proof), using the Littlewood-Paley functions $\tilde{\psi}_{j}, j \in \mathbb{Z}$, introduced above:

$$
f g=T_{f} g+T_{g} f+R(f, g)
$$

where

$$
T_{f} g=\sum_{j \in \mathbb{Z}} \tilde{\Psi}_{j-3}(D) f \tilde{\psi}_{j}(D) g, \quad R(f, g)=\sum_{j \in \mathbb{Z}} \sum_{i=j-3}^{j+3} \tilde{\psi}_{i}(D) f \tilde{\psi}_{j}(D) g,
$$

and we have set $\tilde{\Psi}_{j}(D)=\sum_{i \leq j} \tilde{\psi}_{i}(D)$. We apply this "paraproduct" to $\partial_{\alpha} u \partial_{\beta} u$ :

$$
\partial_{\alpha} u \partial_{\beta} u=T_{\left(\partial_{\alpha} u\right)}\left(\partial_{\beta} u\right)+T_{\left(\partial_{\beta} u\right)}\left(\partial_{\alpha} u\right)+R\left(\partial_{\alpha} u, \partial_{\beta} u\right) .
$$

We will bound $T_{\left(\partial_{\alpha} u\right)}\left(\partial_{\beta} u\right)$ and $R\left(\partial_{\alpha} u, \partial_{\beta} u\right)$ separately. We start with the highfrequency component and write:

$$
\begin{aligned}
R\left(\partial_{\alpha} u, \partial_{\beta} u\right) & =\sum_{j}\left(\sum_{i=j-3}^{j+3} 2^{i} \sigma_{i}^{\alpha}(D) u\right) \tilde{\psi}_{j}(D)\left(\partial_{\beta} u\right) \\
& \approx \sum_{j}\left(\sum_{i=j-3}^{j+3} \sigma_{i}^{\alpha}(D) u\right) \rho_{j}^{\beta}(D)(\Delta u),
\end{aligned}
$$

where $\sigma_{i}^{\alpha}(D)=\sigma^{\alpha}\left(2^{-i} D\right)$ with

$$
\sigma^{\alpha}(k)=\tilde{\psi}(k) k_{\alpha},
$$

and, similarly, $\rho_{j}^{\beta}(D)=\rho^{\beta}\left(2^{-j} D\right)$ with

$$
\rho^{\beta}(k)=\tilde{\psi}(k) k_{\beta}|k|^{-2} .
$$

Above, we have used that $|k| \sim 2^{j}$ on $\operatorname{Supp} \tilde{\psi}_{j}$. In particular, both $\sigma^{\alpha}$ and $\rho^{\beta}$ are functions in $\mathcal{S}\left(\mathbb{R}^{3}\right)$ vanishing in a neighborhood of the origin (given the support properties of $\tilde{\psi}$ ). Hence, we can apply the theory developed by Coifman and Meyer in [CM78] (see also [Tay91], page 90), and conclude that:

$$
\left\|R\left(\partial_{\alpha} u, \partial_{\beta} u\right)\right\|_{L^{2}} \leq C_{1}\|u\|_{\text {BMO }}\|\Delta u\|_{L^{2}} .
$$

We now consider the low-frequency part and proceed in an analogous fashion, writing

$$
\begin{aligned}
T_{\left(\partial_{\alpha} u\right)}\left(\partial_{\beta} u\right) & =\sum_{j}\left(\sum_{i \leq j-5} \tilde{\psi}_{i}(D)\left(\partial_{\alpha} u\right)\right) \tilde{\psi}_{j}(D)\left(\partial_{\beta} u\right) \\
& =\sum_{j} 2^{-j}\left(\sum_{i \leq j-5} \tilde{\psi}_{i}(D)\left(\partial_{\alpha} u\right)\right) \rho_{j}^{\beta}(D)(\Delta u) \\
& =\sum_{j} 2^{-j}\left(\sum_{i \leq j-5} 2^{i} \sigma_{i}^{\alpha}(D) u\right) \rho_{j}^{\beta}(D)(\Delta u) \\
& =\sum_{j}\left(\sum_{i \leq j-5} 2^{-(j-i)} \sigma_{i}^{\alpha}(D) u\right) \rho_{j}^{\beta}(D)(\Delta u),
\end{aligned}
$$


Again we can apply the theory in [CM78], so that we also have

$$
\left\|T_{\left(\partial_{\alpha} u\right)}\left(\partial_{\beta} u\right)\right\|_{L^{2}} \leq C_{2}\|u\|_{\mathrm{BMO}}\|\Delta u\|_{L^{2}} .
$$

The desired estimate then follows from (3.7).

We turn now to the proof of Theorem 1, which consists in first obtaining a continuation principle for strong solutions and then in applying it to weak solutions. We are going to sketch the main ideas and refer to [KT00] for details.

\section{Sketch of proof.}

Step 1. Since $u \in L^{2}\left([0, T], H^{3 / 2}\right)$, for each $\epsilon>0$ there exists $t_{0} \in(0, \epsilon)$ such that $u\left(t_{0}\right) \in H^{s}, s \leq 3 / 2$. Then, as long as $f \in L^{r}\left([0, T], L^{2}\right)$, a short-time strong solutions in $C\left(\left[\epsilon, T_{*}\right], H^{s}\right) \cap C\left(\left(\epsilon, T_{*}\right], H^{1}\right)$, with $s \in(1 / 2,1)$, can be obtained by applying the Kato method (see e.g. [Kat83],[Can95]); that is, by finding fixed-points of the associated Volterra-type integral equation

$$
u(t)=e^{\nu t \Delta} u_{0}-\int_{0}^{t} e^{\nu(t-s) \Delta}[\mathbb{P} \operatorname{div}(u \otimes u)(s)-f(s)] d s,
$$

where $\mathbb{P}$ is the Leray projection onto the space of divergence-free vector fields. The time of existence $T_{*} \leq T$ depends only on the size of the initial data, the size of $f$ in $L^{r}\left([0, T], L^{2}\right)$, and $\nu$. Then, for fixed $\nu$, continuation for the solution is obtained via Gronwall's inequality, once a priori exponential bounds on the $H^{1}$ norm of $u(t)$, $0<t<T$, are established using (3.2), as long as $\|f\|_{L^{r}\left([0, T], L^{2}\right)}$ stays finite. We follow Kozono-Taniuchi and derive an equation for $v=\partial_{i} u, i=1, \ldots, 3$ :

$$
\partial_{t} v-\nu \Delta v+u \cdot \nabla v \nabla q=F+\partial_{i} f
$$

where $F=-\partial_{i} u \cdot \nabla u$ and $q=\partial_{i} p$.

Then, multiplying (3.11) by $v$ and integrating gives for every $0<t_{0} \leq t \leq T$,

$$
\begin{aligned}
\|v(t)\|_{L^{2}}^{2}+2 \nu & \int_{t_{0}}^{t}\|\nabla v(\tau)\|_{L^{2}}^{2} d \tau \leq\left\|v\left(t_{0}\right)\right\|_{L^{2}}^{2}+ \\
& +2 \int_{t_{0}}^{t}\|f(\tau)\|_{L^{2}}\|\nabla v(\tau)\|_{L^{2}} d \tau+2 \int_{t_{0}}^{t}\|F(\tau)\|_{L^{2}}\|v(\tau)\|_{L^{2}} d \tau .
\end{aligned}
$$

By Cauchy's inequality, we obtain

$$
\begin{aligned}
\|v(t)\|_{L^{2}}^{2}+2 \nu \int_{t_{0}}^{t} \| \nabla v & (\tau)\left\|_{L^{2}}^{2} d \tau \leq\right\| v\left(t_{0}\right)\left\|_{L^{2}}^{2}+\nu^{-1} \int_{t_{0}}^{t}\right\| f(\tau) \|_{L^{2}}^{2} d \tau+ \\
& \quad \nu \int_{t_{0}}^{t}\|\nabla v(\tau)\|_{L^{2}}^{2} d \tau+2 \int_{t_{0}}^{t}\|F(\tau)\|_{L^{2}}\|v(\tau)\|_{L^{2}} d \tau .
\end{aligned}
$$

We now use (3.6) and again Cauchy's inequality:

$$
\begin{aligned}
\|v(t)\|_{L^{2}}^{2} & +\frac{\nu}{2} \int_{t_{0}}^{t}\|\nabla v(\tau)\|_{L^{2}}^{2} d \tau \leq \\
& \leq\left\|v\left(t_{0}\right)\right\|_{L^{2}}^{2}+\nu^{-1} \int_{t_{0}}^{t}\|f(\tau)\|_{L^{2}}^{2} d \tau+\frac{\bar{C}^{2}}{2} \nu^{-1} \int_{t_{0}}^{t}\|u(\tau)\|_{H^{3 / 2}}^{2}\|v(\tau)\|_{L^{2}}^{2} d \tau .
\end{aligned}
$$


Finally, it follows from Grönwall's inequality,

$$
\begin{aligned}
& \sup _{t_{0}<t \leq T}\|u\|_{H^{1}} \leq\left(\left\|u\left(t_{0}\right)\right\|_{H^{1}}^{2}+\frac{\nu^{-1}}{2} \int_{t_{0}}^{T}\right.\left.\|f(\tau)\|_{L^{2}}^{2} d \tau\right)^{1 / 2} . \\
& \cdot \exp \left(\frac{\bar{C}^{2}}{2} \nu^{-1} \int_{t_{0}}^{t}\|u(\tau)\|_{H^{3 / 2}}^{2} d \tau\right) .
\end{aligned}
$$

Step 2. We prove that weak solutions satisfying (3.2) fulfill the energy identity:

$$
\|u(t)\|_{L^{2}}^{2}+2 \nu \int_{t_{0}}^{t}\|\nabla u(\tau)\|_{L^{2}}^{2} d \tau=\left\|u\left(t_{0}\right)\right\|_{L^{2}}^{2}+2 \int_{t_{0}}^{t}(f(\tau), u(\tau)) d \tau .
$$

We use the proof of Lemma 3 in [KT00] and we need only show that

$$
\int_{t_{0}}^{t}\left(f(\tau), u_{h}(\tau)\right) d \tau \rightarrow \int_{t_{0}}^{t}(f(\tau), u(\tau)) d \tau
$$

where $u_{h}$ is a certain sequence of test functions converging strongly to $u$ in $L^{2}\left([0, T], H^{1}\right)$. But then (3.14) follows from the regularity assumption on $f$. In particular, Lemma 2 implies that $\left(u \cdot \nabla u, u_{h}\right) \rightarrow(u \cdot \nabla u, u)=0$.

Next, we establish uniqueness of weak solutions with regularity (3.2). Let $u, v$ be weak solutions on $\mathbb{R}^{3} \times[0, T]$ both having initial data $u_{0}$, such that $u \in$ $L^{2}\left([0, T], H^{3 / 2}\right)$ and $v$ satisfies the energy inequality

$$
\|v(t)\|_{L^{2}}^{2}+2 \nu \int_{t_{0}}^{t}\|\nabla v(\tau)\|_{L^{2}}^{2} d \tau \leq\left\|v\left(t_{0}\right)\right\|_{L^{2}}^{2}+2 \int_{t_{0}}^{t}(f(\tau), v(\tau)) d \tau .
$$

We employ the divergence-free test function $u_{h}$ and $v_{h}^{k}$ in [KT00] (following Masuda [Mas84]), where $u_{h}, v_{h}^{k}$ converge strongly to $u$ and $v$ in $L^{2}\left([0, T], H^{1}\right)$. Passing to the limits $k \rightarrow \infty, h \rightarrow 0$, we derive the following equations for $u$ and $v$ :

$$
\begin{aligned}
& \left(\partial_{t} u, v\right)+(u \cdot \nabla u, v)+\nu(\nabla u, \nabla v)=(f, v), \\
& \left(\partial_{t} v, u\right)+(v \cdot \nabla v, u)+\nu(\nabla v, \nabla u)=(f, u),
\end{aligned}
$$

where we suppressed the $t$ dependence for simplicity. We then sum the two equations above and integrate by parts:

$$
\frac{d}{d t}(u, v)+2 \nu(\nabla u, \nabla v)+(v \cdot \nabla v, u)-(u \cdot \nabla v, u)=(f, u+v),
$$

from which we obtain

$$
\begin{aligned}
(u(t), v(t))-\left\|u_{0}\right\|_{L^{2}}^{2} & +\int_{0}^{t}[2 \nu(\nabla u(\tau), \nabla v(\tau))+(v(\tau) \cdot \nabla v(\tau), u(\tau)) \\
& -(u(\tau) \cdot \nabla v(\tau), u(\tau))] d \tau=\int_{0}^{t}(f(\tau),(u+v)(\tau)) d \tau
\end{aligned}
$$


But $u$ satisfies (3.13), while (3.15) holds for $v$. We therefore multiply (3.16) by -2 and sum it to (3.13) and (3.15) to conclude that

$$
\begin{aligned}
\|u(t)-v(t)\|_{L^{2}}^{2}+2 \nu \int_{0}^{t}\|\nabla u(\tau)-\nabla v(\tau)\|_{L^{2}}^{2} d \tau & \\
-2 \int_{0}^{t}((v(\tau)-u(\tau)) \cdot \nabla v(\tau), u(\tau)) d \tau \leq 2 & \int_{0}^{t}(f(\tau),(u+v)(\tau)) d \tau \\
& -2 \int_{0}^{t}(f(\tau),(u+v)(\tau)) d \tau,
\end{aligned}
$$

as the initial data for $u$ and $v$ are the same. Note that the terms containing $f$ exactly cancel out. Hence a differential inequality for $w=v-u$ holds, as in [KT00]:

$$
\|w(t)\|_{L^{2}}^{2}+2 \nu \int_{0}^{t}\|\nabla w(\tau)\|_{L^{2}}^{2} d \tau \leq 2 \int_{0}^{t}(w(\tau) \cdot \nabla v(\tau), u(\tau)) d \tau, \quad \forall 0<t<T .
$$

Finally, we can conclude that $w(t) \equiv 0$ for any $0 \leq t \leq T$, observing that

$$
(w \cdot \nabla v, u)=(w \cdot \nabla w, u)+(w \cdot \nabla u, u)=(w \cdot \nabla w, u),
$$

since $w$ is divergence-free and $u$ satisfies $(3.2)$, so that $(w \cdot \nabla u, u)=0$. In fact, $\forall 0<t<T$,

$$
\begin{aligned}
\|w(t)\|_{L^{2}}^{2}+2 \nu \int_{0}^{t}\|\nabla w(\tau)\|_{L^{2}}^{2} d \tau & \leq 2 \int_{0}^{t}(w(\tau) \cdot \nabla w(\tau), u(\tau)) d \tau \\
& \leq 2 \int_{0}^{t}\|w(\tau)\|_{L^{2}}\|\nabla w(\tau)\|_{L^{2}}\|u(\tau)\|_{H^{3 / 2}} d \tau \\
& \leq \frac{\nu}{2} \int_{0}^{t}\|\nabla w(\tau)\|_{L^{2}}^{2} d \tau \\
& +4 \nu^{-1} \int_{0}^{t}\|w(\tau)\|_{L^{2}}^{2}\|u(\tau)\|_{H^{3 / 2}}^{2} d \tau
\end{aligned}
$$

by Young's inequality. The bound

$$
\|u \cdot \nabla w\|_{L^{2}} \leq C\|u\|_{H^{3 / 2}}\|\nabla w\|_{L^{2}},
$$

used above can be established by arguments similar to those of Lemma 2 .

Combining the uniqueness result for weak solutions with the continuation principle for strong solutions given by (3.12) finishes the proof.

Remark 3.1. Existence and uniqueness of solutions in the class $C\left([0, T], H^{s}\right), s \in$ $(1 / 2,1)$ can be obtained under weaker conditions on the forcing term, namely $f \in$ $L^{r}\left([0, T], L^{2}\right)$ with $r>4 / 3$ depending on $s$. Also, we only need $f \in L^{2}\left([0, T], L^{2}\right)$ for the Grönwall estimate (3.12). The higher regularity on $f$ is used to establish existence of strong solutions in $C\left([0, T], H^{1}\right)$. A "smallness" condition on the norm of $u$ in $L^{2}\left([0, T], H^{3 / 2}\right)$ seems necessary in order to obtain solutions with forcing $f \in L^{4 / 3}\left([0, T], L^{2}\right)$. Due to this gap in regularity for $f$, Theorem 1 cannot be viewed as a truly local condition on weak solutions. Indeed, if a Leray-Hopf solution is localized to a ball, then the forcing introduced into the equation belongs exactly to $L^{4 / 3}\left([0, T], L^{2}\right)$ (see $[\mathrm{Koz} 98]$ and Section 4 below). 
Remark 3.2. The a priori bound (3.1) is the weakest condition that can be imposed given the regularity criteria $(\mathrm{K}-\mathrm{T})$ and $(\mathrm{BdV})$. In fact, $H^{s} \nsubseteq \mathrm{BMO}$ when $s<$ $3 / 2$. In addition, $L^{2}\left([0, T], H^{3 / 2}\right)$ is the only space with norm invariant under the parabolic rescaling of NS in the class $L^{2}\left([0, T], H^{s}\right), s \in \mathbb{R} . L^{2}([0, T], \mathrm{BMO})$ and $L^{2}\left([0, T], W^{1,3}\right)$ are also invariant under the same dilations. Here, by dilations we mean local dilations with factor $\lambda \leq 1$.

We also note that in two dimensions the scale-invariant norm is $L^{2}\left((0, T), H^{1}\left(\mathbb{R}^{2}\right)\right)$, and the critical rate of decay for the energy spectrum becomes $\bar{E}(\kappa)=O\left(\kappa^{-3}\right)$, that is, the rate of the Kraichnan spectrum (cf. [Con97]). Of course, Leray-Hopf weak solutions, which belong to $L^{2}\left((0, T), H^{1}\right)$, are regular in dimension 2 .

Given that the regularity criteria (3.2) is really a continuation principle for strong solutions, any solution developing a singularity at $t=T$ will have infinite $L^{2}\left([0, T], H^{3 / 2}\right)$ norm. The purpose of the next section is to investigate the sharpness of condition (3.1) with respect to formation of singularities in the solution. More precisely, we show that for (hypothetical) self-similar singularities at an isolated point in space and time, the $H^{3 / 2}$ norm grows exactly like $1 / \sqrt{T-t}$ as $t \rightarrow T_{-}$.

\section{Self-Similar SOlutions}

Let $u$ be a weak solution of the Navier-Stokes equation on $D \times[0, T]$. We assume that $u$ has an isolated singularity in space-time. Without loss of generality, the singular point is at $x=0$ and $t=T$, and the solution continues as a weak solution for $T^{\prime}>T$. The rescaled function $\lambda u\left(\lambda x, \lambda^{2}(t-T)+T\right), \lambda \neq 0$, is also a solution, and for $\lambda$ small it gives the asymptotic behavior of $u$ near the singularity. In the limit $\lambda \rightarrow 0$, if such limit exists, $u_{\lambda} \rightarrow \bar{u}$, where $\bar{u}$ is a self-similar solutions of NS, that is,

$$
\bar{u}(x, t)=\lambda \bar{u}\left(\lambda x, \lambda^{2}(t-T)+T\right) .
$$

In particular, $u$ has a well-defined profile at the singular time $T$. So, in some sense $u$ has the simplest possible singularity, although it is not known for the Navier-Stokes equation whether isolated singularities (if they exist) are always self-similar.

The scaling invariance of Navier-Stokes implies a certain relation between the size of a solution at a certain scale and the scale itself. This observation justifies looking for solutions in the form:

$$
u(x, t)=\frac{1}{\lambda(t)} U\left(\frac{x}{\lambda(t)}, \tau\right),
$$

where $\lambda(t) \sim \sup _{x}|u(x, t)|^{-1}$ (so that $U$ is bounded), and $\tau$ is related to $t$ by:

$$
d \tau=\frac{1}{\lambda(t)^{2}} d t
$$

This approach corresponds to a dynamic rescaling of the equation. In the selfsimilar case, $\lambda(t)=\sqrt{T-t}$, so that $\tau=\log \frac{T}{T-t}$, and

$$
u(x, t)=\frac{1}{\sqrt{T-t}} U\left(\frac{x}{\sqrt{T-t}}, \tau\right) .
$$


$U(y, \tau)$ is a solution of:

$$
\left\{\begin{array}{l}
\partial_{\tau} U+\frac{1}{2}(U+y \cdot \nabla U)+U \cdot \nabla U+\nabla P-\nu \Delta U=0 \\
\operatorname{div} U=0
\end{array}\right.
$$

The so-called Leray backward solutions correspond to $\partial_{\tau} U=0$. It is known there are no non-trivial Leray backward solutions satisfying the energy inequality, even locally, [NRŠ96],[Tsa98]. However, by comparison with the complex GinzburgLandau equation, which has the same scaling law and energy identity as NavierStokes, we can conjecture the existence of periodic solutions to (4.4) [PŠ01]. In fact, we can then view (4.4) as a Navier-Stokes equation with a forcing periodic in $\tau$.

The most natural behavior at infinity for self-similar solutions, which is for example consistent with partial regularity (see [Tsa98]), is given by:

$$
u(x, t) \sim \frac{1}{|x|} v\left(\frac{x}{|x|}, t\right)+o\left(\frac{1}{|x|}\right), \quad x \rightarrow \infty,
$$

where $v(\cdot): S^{2} \times[0, T] \rightarrow \mathbb{R}^{3}$. We therefore choose $U$ to have the behavior

$$
U(y, \tau)=\frac{1}{|y|} A\left(\frac{1}{|y|}, \tau\right),
$$

for $|y| \leq R^{\prime}, R^{\prime}$ large enough, with $A: S^{2} \times \mathbb{R} \rightarrow \mathbb{R}^{3}$ smooth, periodic in $\tau$. The profile at the blow-up time is then a homogeneous function of degree -1 . We remark that there are no weak solutions to NS that are locally homogeneous of degree $\alpha<-1$ [CP01].

Following arguments in [Tsa98] we can prove that a solution $u$ of the form (4.3), where $U$ satisfies (4.6), is a suitable weak solution in the sense of Caffarelli-KohnNirenberg [CKN82]. First of all, a lengthy but straightforward calculation shows that $\mathrm{u}$ satisfies the local energy estimate:

$$
\text { ess } \sup _{t_{1}<t \leq T} \int_{B} \frac{1}{2}|u(x, t)|^{2} d x+\int_{t_{1}}^{T} \int_{B} \nu|\nabla u(x, t)|^{2} d x d t<\infty,
$$

where $B$ is a ball in $\mathbb{R}^{3}$ and $t_{1}<T$. Moreover, since $u$ is smooth for $t<T$, it satisfies the generalized energy inequality on any cylinder $B \times\left(t_{1}, T\right)$. Therefore, it is enough to establish that the pressure $p$ belongs to $L^{5 / 4}\left(B \times\left(t_{1}, T\right)\right)$. This estimate in turn follows exactly as in [Tsa98] (Section 4) for the steady case. In fact, the necessary ingredients in the proof are the decay $U(y)=O(1 /|y|)$ at infinity (which is assumed here) and regularity results in the pressure for weak solutions to the Stokes system with forcing $\frac{1}{2}(U+y \cdot \nabla U)+U \cdot \nabla U$ (see [Gal94] page 180). In our case, there is an extra term $\frac{\partial U}{\partial \tau}$ in the forcing, but $\frac{\partial U}{\partial \tau}$ has the same regularity and decay as $U$ given (4.6) and the smoothness of $U$.

The solution $u$ described above is a weak solutions to the Navier-Stokes equation in the sense of Definition 2.1 on any space-time cylinder $D \times[0, T]$, where $D$ is an open, bounded subset of $\mathbb{R}^{3}$, but not on the whole space. Since we are interested in the behavior near the singularity (or equivalently in the high wavenumber asymptotics), we are then going to "localize" NS to a large ball $B(0, R)$ centered at the origin with radius $R$. To this end, let $\eta \in C_{0}^{\infty}$ be a cut-off function such that $\eta \equiv 1$ on $B(0, R)$, Supp $\eta \subset B(0, R+1)$. Without loss of generality, we can arrange for $R=R^{\prime}$, and we can take $\eta$ radial. The "localized" solution $\tilde{u}$ is the divergence-free part of $\eta u$. Using Bogovski's theorem, it is possible to construct a vector field $U$, 
supported on $B(0, R)$, such that $\operatorname{div} U=\operatorname{div}(\eta u)$; then the equations for $U$ are truly local in $B(0, R)$ as in [Koz98]. We only need a control on the behavior of the solution at infinity, therefore we apply the Hodge decomposition in the whole $\mathbb{R}^{3}$ :

$$
\tilde{u}=\eta u+\nabla g, \quad \operatorname{div} \tilde{u}=0 .
$$

By Hodge's theorem (see, e.g., [Gal94]), $\tilde{u}$ has the same regularity and decay properties as $\eta u . \tilde{u}$ is now a weak solution in the sense of Definition 2.1 in $\mathbb{R}^{3} \times[0, T]$ of:

$$
\partial_{t} \tilde{u}-\nu \Delta \tilde{u}+\tilde{u} \cdot \nabla \tilde{u}+\nabla \tilde{p}=\tilde{f} .
$$

with forcing $\tilde{f}$ given by:

$$
\tilde{f}(u, p, \eta)=\left(\eta^{2}-\eta\right) u \cdot \nabla u-\nu u \Delta \eta+p \nabla \eta-2 \nu \nabla \eta \cdot \nabla u-\eta u \cdot \nabla^{2} g-\nabla g \cdot \nabla(\eta u),
$$

where $p$ is the pressure function associated to $u$. Above, we have included in $\nabla \tilde{p}$ all the terms that are gradients.

To compare the decay rate of the energy spectrum of $\tilde{u}$ with the results of Theorem 1 , it is necessary to ensure that $\tilde{f} \in L^{r}\left([0, T], L^{2}\left(\mathbb{R}^{3}\right)\right)$ for some $r>2$. We first observe that except for $\eta u \cdot \nabla^{2} g+\nabla g \cdot \nabla(\eta u)$, all the other terms are supported in the shell $D_{R}=\{x|R<| x \mid<R+1\}$. If $t$ is close enough to $T$, we can assume that $U$ has the form (4.6) for any $x$ in $D_{R}$. Then, an explicit simple calculation shows that $u \in C\left([0, T], C^{k}\left(D_{R}\right)\right)$, for every $k$. Also, the pressure function $p$ is given for $0 \leq t<T$ by

$$
p(x, t)=\frac{1}{T-t} P\left(\frac{x}{T-t}, \tau\right),
$$

where $P$ solves for each fixed $\tau$

$$
\Delta P=\operatorname{div}(\operatorname{div} U \otimes U) .
$$

In particular, $P$ is smooth in $y$ and $\tau$. Then, since $U(y, \tau)=O(1 /|y|)$ as $y \rightarrow \infty$, using the methods in [NRŠ96] and [Tsa98], we obtain that

$$
P(y, \tau)=O\left(\frac{1}{|y|^{2-\epsilon}}\right), \quad y \rightarrow \infty,
$$

for $\epsilon$ arbitrarily small. Combining (4.10) with this decay estimate we can conclude that $p \in L^{r}\left([0, T], L^{2}\left(D_{R}\right)\right)$, by taking $\epsilon$ small enough.

To control $\eta u \cdot \nabla^{2} g+\nabla g \cdot \nabla(\eta u)$ near the singularity, we remark that $g$ is harmonic on $B(0, R)$, since $\eta u=u$ there, and therefore it is smooth near the origin. Therefore it is enough to prove that $\eta u \in L^{r}\left([0, T], H^{1}\right)$, for $r>2$ but close to 2 . We will do so by showing first that $\eta u \in L^{2, \infty}\left([0, T], H^{3 / 2}\right)$ and interpolating.

Remark 4.1. If $u$ is a weak solutions to NS on space-time cylinders $D \times[0, T]$ such that $u$ is -1 homogeneous at infinity, not necessarily self-similar, we can still conclude that $\tilde{f} \in L^{r}\left([0, T], L^{2}\right)$, for $4 / 3<r<2$. Then, if $u$ satisfies (3.2), by Theorem $1 \tilde{u}$ belongs to $C\left([0, T], H^{1 / 2+\epsilon}\right)$ for $\epsilon>0$ small.

We make some preliminary remarks. First of all, as we have already noted, it is sufficient to estimate the $H^{3 / 2}$-norm of $\eta u(t)$ in for $t \in[\bar{t}, T), \bar{t}$ close to $T$. As $t \rightarrow T$, the behavior of $\eta u(t)$ becomes the behavior of $U$ in a neighborhood of infinity, which is essentially the complement of the ball $B(0, R / \sqrt{T-t})$. Heuristically, 
since $\hat{U}(k, \tau)$ is homogeneous of degree -1 near the origin, $\widehat{\eta u(t)}(k)$ is homogeneous of degree -2 up to frequencies $|k| \sim 1 / \sqrt{T-t}$, while it decays fast at larger frequencies, given that $\eta u(t)$ is smooth for $t<T$. Consequently:

$$
\|\eta u(\cdot, t)\|_{H^{3 / 2}}^{2}=\int_{\mathbb{R}^{3}}|k|^{3}|\widehat{\eta u(t)}(k)|^{2} d k \approx \int_{0}^{1 / \sqrt{T-t}} \kappa d \kappa \approx \frac{1}{T-t} .
$$

We make this argument precise in the next proposition. We cannot use dilations directly, as $\eta u$ is only approximately homogeneous and only at time $t=T$. Moreover, $u$ depends on $t$ both through the self-similar variable and $\tau$. We find therefore convenient to employ a wavelet characterization of $H^{3 / 2}$, which naturally lends itself to rescaling and localization.

We consider an $L^{2}$-orthogonal basis $W=W_{1} \cup W_{2}$ of compactly supported wavelets, normalized in $L^{1}$, where

$$
\begin{array}{ll}
W_{1}=\left\{2^{3 j} \psi_{\epsilon}\left(2^{j} x-k\right)\right\} & j \in \mathbb{N}, k \in \mathbb{Z}^{3}, \epsilon=1, \ldots, 7, \\
W_{2}=\{\phi(x-k)\} & k \in \mathbb{Z}^{3} .
\end{array}
$$

For a construction of this basis we refer, for example, to [Mey92]. To simplify notation, we suppress the dependence on $\epsilon$. We call $\phi$ the "father wavelet" and $\psi$ the "mother wavelet". We denote by $\phi_{k}$ and $\psi_{j, k}$ their translated and dilated versions.

$W$ is chosen to give a multiresolution of $L^{2}$ of regularity $2=r>3 / 2$, which in particular means $\psi$ has exactly the first three vanishing moments. We require the wavelets to have the minimal smoothness necessary to resolve $H^{3 / 2}$, so that their support is the smallest possible (see e.g. [HW96]).

The $H^{3 / 2}$ norm is then expressed entirely in terms of wavelets coefficients as

$$
\|f\|_{H^{3 / 2}}^{2}=\sum_{k \in \mathbb{Z}^{3}}\left|\left(\phi_{k}, f\right)\right|^{2}+\sum_{j \in \mathbb{N}} \sum_{k \in \mathbb{Z}^{3}}\left|\left(\psi_{j, k}, f\right)\right|^{2} .
$$

A proof can be found again in [Mey92]. The integer vectors $k$ should be thought as "locations", while the integer $j$ as "scales", since the wavelets $\psi_{j, k}$ are supported on (small) multiples of dyadic cubes $\mathrm{C}_{j, k}$, with length $2^{j}$ and lower corner $2^{-j} k$.

Proposition 3. Let $u$ be of the form (4.3) with $U$ satisfying (4.6). Then, as $t \rightarrow T$,

$$
\|\eta u(\cdot, t)\|_{H^{3 / 2}}^{2}=\frac{1}{T-t} G(\tau)+o\left(\frac{1}{T-t}\right),
$$

where $G$ is a positive, periodic function of $\tau$.

Proof. We first observe that the cut-off function $\eta$ simply limits the allowed $k^{\prime} s$ at each scale in the wavelet decomposition of $u(t)$, since $\eta$ is time-independent. In fact, for a fixed $j$, except for a finite number of locations $k, \eta \psi_{j, k}=\psi_{j, k}$ or else it vanishes identically (and similarly for $\eta \phi_{k}$ ), as we consider only cubes of size 1 or smaller. The exceptional locations are precisely those corresponding to cubes on the shell $R<|x|<R+1$. Therefore,

$$
\|\eta u(\cdot, t)\|_{H^{3 / 2}}^{2} \approx\left(\sum_{|k| \leq R}\left|\left(\phi_{k}, u(t)\right)\right|^{2}\right)+\left(\sum_{j \geq 0} \sum_{|k|<R 2^{j}}\left|\left(\psi_{j, k}, u(t)\right)\right|^{2}\right) .
$$

We now rescale variables $y=\frac{x}{\sqrt{T-t}}$, so that we can express the wavelets coefficients of $u$ in terms of those of $U$, provided we shift scales appropriately. For fixed 
$t$, define the scale $J>0$ by the formula

$$
J=\left[-\log _{2}(\sqrt{T-t})\right] .
$$

Then,

$$
\begin{aligned}
\left(\psi_{j, k}, u(t)\right) & =2^{2 J} 2^{3 j} \int_{\mathbb{R}^{3}} \psi\left(2^{j} x-k\right) U\left(2^{J} x\right) d x \\
& =2^{2 J} 2^{3(j-J)} \int_{\mathbb{R}^{3}} \psi\left(2^{j-J} y-k\right) U(y) d y \\
& =2^{J}\left(\psi_{j-J, k}, U\right)=2^{J} c(j-J, k),
\end{aligned}
$$

and similarly $\left(\phi_{k}, u(t)\right)=2^{J}\left(\phi_{-J, k}, U\right)=2^{J} a(k)$. Note the $k$ 's do not change. Consequently,

$$
\|\eta u(\cdot, t)\|_{H^{3 / 2}}^{2} \approx 2^{2 J}\left(\sum_{0 \leq|k|<R}|a(k)|^{2}\right)+2^{2 J}\left(\sum_{\bar{j} \geq-J} \sum_{0 \leq|k|<R 2^{\bar{j}+J}}|c(\bar{j}, k)|^{2}\right),
$$

where $\bar{j}=j-J$. So, $\bar{j} \rightarrow-\infty$, as $t \rightarrow T$.

Above, we have explicitly dropped the dependence on $\tau$, but it is understood that every coefficient is a periodic function in $\tau$.

Next we estimate the wavelet coefficients, starting from $a(k)$, that is, the lowfrequency contribution to (4.13). For $t$ close to $T, J$ is large and $|k| \lesssim R<<2^{J}$, the size of the support of $\phi_{-J, k}$, which can be taken strictly positive near the lower corner of the cube. Therefore we can assume $a(k)$ is essentially constant in $k$, given that $U$ is smooth, so that

$$
\begin{aligned}
|a(k)| & \approx 2^{-3 J}\left|\int_{\mathbb{R}^{3}} \phi\left(2^{-J} y\right) U(y) d y\right| \leq C_{1} 2^{-3 J} \int_{B\left(0, M 2^{J}\right)}|U(y)| d y \\
& \leq C_{2} 2^{-3 J}+C_{2}^{\prime} 2^{-3 J}\left[2^{2 J}-R^{2}\right] \int_{S^{2}}|A(u)| d \sigma(u),
\end{aligned}
$$

where the last inequality follows by splitting the integral as an integral over the ball $B(0, R)$ and an integral over the shell $\left\{R<|y|<R 2^{J}\right\}$. Here $M$ is a small integer. But the sum over $k$ in (4.15) is finite, independently of $t$, so that it contributes at most a factor of order $2^{-2 J}$ and we can conclude that

$$
2^{2 J} \sum_{0 \leq|k|<R}|a(k)|^{2}=O(1) .
$$

As for the contribution from the $c(\bar{j}, k)$, that is the high-frequency part of (4.13), we distinguish two cases, when $\bar{j} \geq 0$ and when $-J \leq \bar{j}<0$.

Case $I, \bar{j} \leq 0$ : We first consider wavelets supported on cubes contained in the ball $B(0, R)$. Since the cubes have size at most of order one, it is equivalent to pick only locations $k$ for which $|k|<2^{\bar{j}} R$ at a fixed scale $\bar{j}$. Therefore, there are $O\left(2^{3 \bar{j}}\right)$ of such cubes. At the same time, the wavelet coefficients $c(\bar{j}, k)$ are rapidly decreasing in $\bar{j}$ because of the regularity of $U$, that is, $|c(\bar{j}, k)| \leq C_{N} 2^{-N \bar{j}}$ for every $N>0$. Consequently,

$$
2^{2 J} \sum_{\bar{j} \geq 0} \sum_{0 \leq|k|<R 2^{\bar{j}}}|c(\bar{j}, k)|^{2}=O\left(2^{2 J}\right) .
$$


Next, we look at wavelets supported on cubes outside the ball $B(0, R)$, which means $2^{\bar{j}} R \leq|k| \leq 2^{\bar{j}+J} R$. For a fixed scale $\bar{j}$, there are still only finitely-many such cubes, but their number grows unboundedly as $t \rightarrow T$. However, we can use the homogeneity of $U$ on $B(0, R)$ to compensate. In fact, since $U$ is homogeneous of degree -1 , a simple change of variables shows that

$$
c(\bar{j}, k)=2^{\bar{j}} c(0, k) .
$$

We estimate $c(0, k)$ using the moment conditions on the wavelets $\psi_{0, k}$. In fact,

$$
c(0, k)=\left(\psi_{0, k}, U\right)=\int_{\mathbb{R}^{3}} \psi(y-k) U(y) d y=\int_{[0, M]^{3}} \psi(\tilde{y}) U(\tilde{y}+k) d \tilde{y},
$$

where $M$ is again some small integer. We then expand $U$ around $k$ in Taylor series, and impose the condition that $\psi$ have the first three vanishing moments:

$$
\begin{aligned}
\int_{[0, M]^{3}} & \psi(\tilde{y}) U(\tilde{y}+k) d \tilde{y}=\int_{[0, M]^{3}} \psi(\tilde{y}) \cdot \\
\cdot & {\left[U(k)+\tilde{y} \cdot \nabla U(k)+\tilde{y} \cdot \nabla^{2} U(k) \cdot \tilde{y}+\left(\int_{0}^{1} \nabla^{3} U(k+\alpha \tilde{y})(\tilde{y}, \tilde{y}, \tilde{y}) \alpha^{3} d \alpha\right)\right] d \tilde{y} } \\
& =\int_{[0, M]^{3}} \psi(\tilde{y})\left(\int_{0}^{1} \nabla^{3} U(k+\alpha \tilde{y})(\tilde{y}, \tilde{y}, \tilde{y}) \alpha^{3} d \alpha\right) d \tilde{y} .
\end{aligned}
$$

But $|k|>>|y|$, and $\nabla^{3} U(k+\tilde{y})$ is homogeneous of degree -4 , in this range. Hence we have

$$
c(0, k) \approx \int_{[0,1]^{3}} \psi(\tilde{y}) \frac{H(\tilde{y})}{|k|^{4}} d \tilde{y}=O\left(|k|^{-4}\right),
$$

where $H$ is a bounded, smooth function. We now evaluate the sum over $k$ and $\bar{j}$ using Euler-Mclaurin formula (cf. [BO99]):

$$
\begin{aligned}
\sum_{2^{\bar{j}} R \leq|k| \leq R 2^{\bar{j}+J}}|c(\bar{j}, k)|^{2} & =\sum_{2^{\bar{j}} R \leq|k| \leq R 2^{\bar{j}+J}} 2^{2 \bar{j}}|c(0, k)|^{2} \\
& \approx C 2^{2 \bar{j}}\left(2^{-\bar{j}} R\right)^{5} \approx C R^{5} 2^{-3 \bar{j}}
\end{aligned}
$$

and finally,

$$
2^{2 J} \sum_{\bar{j} \geq 0} \sum_{2^{\bar{j}}}|c(\bar{j}, k)|^{2}=O\left(2^{2 J}\right) .
$$

Case II: $-J \leq \bar{j}<0$ : Again, we distinguish between wavelets supported near the origin and those far away. Because we are considering negative scales, therefore large cubes, there are only a few cubes with corner in $B(0, R)$, uniformly in $J$. The coefficients $c(\bar{j}, k)$ for these cubes can be bound similarly to the low-frequency components $a(k)$. On the cubes with corners in $B(0, R)^{c}$, we can use homogeneity of $U$ and reduce to $\bar{j}=0$ thank to (4.18). Moreover, $|k| \geq 1$, because the wavelets are supported away from the origin. Consequently, using (4.19),

$$
\sum_{1 \leq|k| \leq R 2^{\bar{j}+J}}|c(\bar{j}, k)|^{2}=\sum_{1 \leq|k| \leq R 2^{\bar{j}+J}} 2^{2 \bar{j}}|c(0, k)|^{2} \approx C 2^{2 \bar{j}},
$$

again by Euler-Mclaurin formula, and (4.21) is replaced by:

$$
2^{2 J} \sum_{-J \leq \bar{j}<0} \sum_{1 \leq|k| \leq R 2^{\bar{j}-J}}|c(\bar{j}, k)|^{2} \approx \sum_{-J \leq \bar{j}<0} 2^{2(J+\bar{j})}=O\left(2^{2 J}\right) .
$$


Estimate (4.12) now follows from (4.16), (4.17), (4.21), and (4.23).

From (4.12), making the change of variables $t=T\left(1-e^{-\tau}\right)$ valid on any subinterval of $[0, T]$, we can conclude that for $\bar{t}<T$, but arbitrarily close to $T$,

$$
\begin{aligned}
& \|\eta u\|_{L^{2}\left([0, t], H^{3 / 2}\right)}^{2} \asymp \sum_{0 \leq n \leq n_{0}} \int_{2(n-1) \pi}^{2 n \pi} F(\tau) d \tau, \\
& \asymp n_{0} \int_{0}^{2 \pi} F(\tau) d \tau \text {, }
\end{aligned}
$$

where $n_{0}=\left[\frac{1}{2 \pi} \log \frac{T}{T-\bar{t}}\right]$; that is,

$$
\|\eta u\|_{L^{2}\left([0, \bar{t}], H^{3 / 2}\right)}^{2} \asymp C(T) \log (T-\bar{t}) .
$$

Therefore $\eta u \in L^{2, \infty}\left([0, T], H^{3 / 2}\right)$. Moreover, $\eta u \in L^{\infty}\left([0, T], L^{2}\right)$, since for $t$ close to $T$

$$
\begin{aligned}
\|\eta u(\cdot, t)\|_{L^{2}}^{2} & =\sqrt{T-t} \int_{\mathbb{R}^{3}} \eta(\sqrt{T-t} x)^{2}|U(x, \tau)|^{2} d x \\
& \asymp \sqrt{T-t} \int_{R}^{R / \sqrt{T-t}} \frac{1}{r^{2}} r^{2} d r .
\end{aligned}
$$

Then, interpolating gives

$$
u \in L^{r}\left([0, T], H^{1}\right),
$$

for some $r>2$. We point out that if the rescaling function $\lambda(t)$ in (4.2) decays slower than $(T-t)^{1 / 2}$, then (3.1) is finite, consistently with blow-up rates near a singularity (cf. [Ler33], [CP01]). We remark, however, that these rates are pointwise estimates of solutions in space and time and they must hold only locally in arbitrarily small space-time cylinders centered at the singularity. In view of these estimates, it is reasonable to expect that $u$ is smooth across the blow-up time $T$ away from the singularity, since $u$ is smooth and small at infinity. This is a rather technical question (which we reserve to address in future work), as we do not have the energy inequality at our disposal and the usual weak-strong results do not apply in this case.

We finally observe that the localized solution $\tilde{u}$ satisfies all the hypothesis of Theorem 1, except the condition on the energy spectrum (3.1). In fact, in the next proposition we quantify precisely the borderline decay of the (local) LittlewoodPaley spectrum $\bar{E}_{L P}$ of $\eta u$ (see (2.4) for the definition).

Proposition 4. Let $u$ and $\eta$ satisfy all the hypotheses of Proposition 3. Then,

$$
\left.\bar{E}(k, t)=O(|k|)^{-4}\right),
$$

for $k \in\left(2^{j-1}, 2^{j+1}\right)$ as $j \rightarrow \infty$.

Proof. Let again $\left\{\psi_{j, k}, \phi_{k}\right\}$ be the wavelet basis used in the proof of Proposition 3, and $\tilde{\psi}_{j}$ be Littlewood-Paley functions. It is not difficult to show that

$$
\left\|\tilde{\psi}_{j}(\cdot) \widehat{\eta u(t)}(\cdot)\right\|_{L^{2}}^{2} \approx \sum_{k \in \mathbb{Z}^{3}} 2^{-3 j}\left|\left(\psi_{j, k}, \eta u(t)\right)\right|^{2},
$$


up to an error that is rapidly decreasing in $j$ (due to the lack of compact support of the wavelets in Fourier space). The factor $2^{-3 j}$ compensates for the wavelet normalization in $L^{1}$, instead of $L^{2}$.

We now fix $j$ large, and define $\bar{t} \in(0, T)$ by the condition

$$
\bar{t}=T-2^{-2 j} .
$$

Given a time $t \in(0, T)$, we let $J$ and $\bar{j}$ be as in the proof of Proposition 3, that is,

$$
\bar{j}=j-J, \quad J=\left[-\log _{2}(\sqrt{T-t})\right] .
$$

We distinguish two cases.

If $t \leq \bar{t}$, then $\bar{j} \geq 0$, and from (4.17), (4.20), we conclude that

$$
\begin{aligned}
E_{L P}(k, t) & \approx 2^{-j}\left\|\tilde{\psi}_{j} \widehat{\eta u(t)}\right\|_{L^{2}}^{2} \approx 2^{-4 j} 2^{2 J} \sum_{0 \leq|k| \leq R 2^{\bar{j}}}|c(\bar{j}, k)|^{2} \\
& \approx N_{1}(\tau) 2^{-4 j} 2^{2 J} 2^{-3(j-J)} \approx N_{1}(\tau) \frac{2^{-7 j}}{(T-t)^{5 / 2}},
\end{aligned}
$$

where $N_{1}$ is smooth and periodic in $\tau$. Recalling that $t=T\left(1-e^{-\tau}\right)$ gives $(4.26)$

$$
\int_{0}^{\bar{t}} E_{L P}(k, t) d t \approx 2^{-7 j} e^{3 \pi n_{0}} \sum_{0 \leq n \leq n_{0}} \int_{2(n-1) \pi}^{2 n \pi} e^{\left(3 \tau / 2-3 \pi n_{0}\right)} N_{1}(\tau) d \tau, \approx \tilde{C}(T) 2^{-4 j},
$$

where again $n_{0}=\left[\frac{1}{2 \pi} \log \frac{T}{T-\bar{t}}\right]$, so that $2^{j}$ and $e^{\pi n_{0}}$ are comparable. If $\bar{t}<t<T$, then $\bar{j}<0$, and from (4.22) we conclude instead that

$$
\begin{aligned}
E_{L P}(k, t) & \approx 2^{-j}\left\|\tilde{\psi}_{j} \widehat{\eta u(t)}\right\|_{L^{2}}^{2} \approx 2^{-4 j} 2^{2 J} \sum_{0 \leq|k| \leq R 2^{\bar{j}+J}}|c(\bar{j}, k)|^{2} \\
& \approx N_{2}(\tau) 2^{-4 j} 2^{2 J} 2^{2(j-J)} \approx N_{2}(\tau) 2^{-2 j},
\end{aligned}
$$

where $N_{2}$ is smooth and periodic in $\tau$. Similarly to (4.26) then:

$\frac{1}{T} \int_{\bar{t}}^{T} E_{L P}(k, t) d t \approx 2^{-2 j} e^{-2 \pi n_{0}} \sum_{n_{0}<n<\infty} \int_{2(n-1) \pi}^{2 n \pi} e^{-\left(\tau-2 \pi n_{0}\right)} N_{2}(\tau) d \tau, \approx \tilde{C}(T) 2^{-4 j}$,

After the blow-up time, one can postulate that the solution continues as a forward self-similar solution. In fact, solutions of this type have been constructed for the harmonic map heat flow (see [Tsa98] for an unpublished reference). On the other hand, the analysis of the complex Ginzburg-Landau equation suggests that the solution is highly oscillating past the singular time [PŠ01]. Nonetheless, forward self-similar solutions with homogeneous initial data exist [GM89],[CMP94],[Can95]. The solution $u$ has the form:

$$
u(x, t)=e^{\nu t \Delta} \bar{u}(x)+w(x, t) .
$$

$w$ should be thought of as a "fluctuation" with respect to the "trend" $e^{\nu t \Delta} \bar{u}(x)$.

Above, $e^{t \Delta}$ is the heat semigroup and $\bar{u}$ is the singular profile, now at $t=0$. The solution is Leray self-similar with

$$
U(x)=e^{\nu \Delta} \bar{u}(x)+W(x) .
$$


The existence of such solutions is again obtained by a fixed-point method in an appropriate Banach space for sufficiently small data, for example if the initial data is -1 homogeneous and its restriction of the unit sphere satisfies

$$
\|\bar{u}\|_{C^{m}\left(S^{2}\right)} \leq \delta, \quad m \geq 1,
$$

$\delta$ a sufficiently small constant. The fluctuation $W$ is smooth on $\mathbb{R}^{3}$ and satisfies the following estimates [CMP94]:

$$
\left|\partial^{\alpha} W(x)\right| \leq C_{\alpha} \frac{1}{(1+|x|)^{2+|\alpha|}}, \quad \forall \alpha,
$$

so that $W$ is in $H^{s}, \forall s \geq 0$. A simple rescaling argument shows again that $u$ belongs to $L^{2, \infty}\left([0,+\infty), H^{3 / 2}\right)$.

\section{REFERENCES}

[BdV95] H. Beirão da Veiga, A new regularity class for the Navier-Stokes equations in $\mathbf{R}^{n}$, Chinese Ann. Math. Ser. B 16 (1995), no. 4, 407-412.

[BO99] C. M. Bender and S. A. Orszag, Advanced mathematical methods for scientists and engineers. I, Springer-Verlag, New York, 1999, Asymptotic methods and perturbation theory, Reprint of the 1978 original.

[Can95] M. Cannone, Ondelettes, paraproduits et Navier-Stokes, Diderot Editeur, Paris, 1995, With a preface by Yves Meyer.

[CKN82] L. Caffarelli, R. Kohn, and L. Nirenberg, Partial regularity of suitable weak solutions of the Navier-Stokes equations, Comm. Pure Appl. Math. 35 (1982), no. 6, 771-831.

[CM78] R. R. Coifman and Y. Meyer, Au delà des opérateurs pseudo-différentiels, Astérisque, vol. 57, Société Mathématique de France, Paris, 1978, With an English summary.

[CMP94] M. Cannone, Y. Meyer, and F. Planchon, Solutions auto-similaires des équations de Navier-Stokes, Séminaire sur les Équations aux Dérivées Partielles, 1993-1994, École Polytech., Palaiseau, 1994, pp. Exp. No. VIII, 12.

[Con97] P. Constantin, The littlemood-paley spectrum in two-dimensional turbulence, Theor. Comput. Fluid Dynam. 9 (1997), no. 3-4, 183-189.

[CP01] Z.-M. Chen and W. G. Price, Blow-up rate estimates for weak solutions of the NavierStokes equations, R. Soc. Lond. Proc. Ser. A Math. Phys. Eng. Sci. 457 (2001), no. $2015,2625-2642$.

[DG95] C. R. Doering and J. D. Gibbon, Applied analysis of the Navier-Stokes equations, Cambridge Texts in Applied Mathematics, Cambridge University Press, Cambridge, 1995.

[DG02] C. R. Doering and J. D. Gibbon, Bounds on moments of the energy spectrum for weak solutions of the three-dimensional Navier-Stokes equations, Phys. D 165 (2002), no. 3-4, 163-175.

[DT95] C. R. Doering and E. S. Titi, Exponential decay rate of the power spectrum for solutions of the Navier-Stokes equations, Phys. Fluids 7 (1995), no. 6, 1384-1390.

[FMRT01] C. Foias, O. Manley, R. Rosa, and R. Temam, Navier-Stokes equations and turbulence, Encyclopedia of Mathematics and its Applications, vol. 83, Cambridge University Press, Cambridge, 2001.

[Fri95] U. Frisch, Turbulence, Cambridge University Press, Cambridge, 1995, The legacy of A. N. Kolmogorov.

[FT79] C. Foiaş and R. Temam, Some analytic and geometric properties of the solutions of the evolution Navier-Stokes equations, J. Math. Pures Appl. (9) 58 (1979), no. 3, 339-368.

[FT89] C. Foias and R. Temam, Gevrey class regularity for the solutions of the Navier-Stokes equations, J. Funct. Anal. 87 (1989), no. 2, 359-369.

[Gal94] G. P. Galdi, An introduction to the mathematical theory of the Navier-Stokes equations. Vol. I, Springer Tracts in Natural Philosophy, vol. 38, Springer-Verlag, New York, 1994, Linearized steady problems.

[Gig86] Y. Giga, Solutions for semilinear parabolic equations in $L^{p}$ and regularity of weak solutions of the Navier-Stokes system, J. Differential Equations 62 (1986), no. 2, 186212. 
[GM89] Y. Giga and T. Miyakawa, Navier-Stokes flow in $\mathbf{R}^{3}$ with measures as initial vorticity and Morrey spaces, Comm. Partial Differential Equations 14 (1989), no. 5, 577-618.

[HW96] E. Hernández and G. Weiss, A first course on wavelets, Studies in Advanced Mathematics, CRC Press, Boca Raton, FL, 1996, With a foreword by Yves Meyer.

[JN61] F. John and L. Nirenberg, On functions of bounded mean oscillation, Comm. Pure Appl. Math. 14 (1961), 415-426.

[Kat83] T. Kato, Quasilinear equations of evolution in nonreflexive Banach spaces, Nonlinear partial differential equations in applied science (Tokyo, 1982), North-Holland Math. Stud., vol. 81, North-Holland, Amsterdam, 1983, pp. 61-76.

[Koz98] H. Kozono, Removable singularities of weak solutions to the Navier-Stokes equations, Comm. Partial Differential Equations 23 (1998), no. 5-6, 949-966.

[KT00] H. Kozono and Y. Taniuchi, Bilinear estimates in BMO and the Navier-Stokes equations, Math. Z. 235 (2000), no. 1, 173-194.

[Ler33] J. Leray, Etude de diverses équations intégrales nonlinéaires et de quelques problémes que pose l'hydrodynamique, J. Math. Pures Appl. 12 (1933), 1-82.

[Lin98] Fanghua Lin, A new proof of the Caffarelli-Kohn-Nirenberg theorem, Comm. Pure Appl. Math. 51 (1998), no. 3, 241-257.

[LR99] P. G. Lemarié-Rieusset, Solutions faibles d'énergie infinie pour les équations de Navier-Stokes dans $\mathbf{R}^{3}$, C. R. Acad. Sci. Paris Sér. I Math. 328 (1999), no. 12, 11331138.

[Mas84] K. Masuda, Weak solutions of Navier-Stokes equations, Tohoku Math. J. (2) 36 (1984), no. $4,623-646$.

[Maz03] A. L. Mazzucato, Besov-Morrey spaces: function space theory and applications to non-linear PDE, Trans. Amer. Math. Soc. 355 (2003), no. 4, 1297-1364.

[Mey92] Y. Meyer, Wavelets and operators, Cambridge Studies in Advanced Mathematics, vol. 37, Cambridge University Press, Cambridge, 1992, Translated from the 1990 French original by D. H. Salinger.

[NRŠ96] J. Nečas, M. Růžička, and V. Šverák, On Leray's self-similar solutions of the NavierStokes equations, Acta Math. 176 (1996), no. 2, 283-294.

[PŠ01] P. Plecháč and V. Šverák, On self-similar singular solutions of the complex GinzburgLandau equation, Comm. Pure Appl. Math. 54 (2001), no. 10, 1215-1242.

[Sch76] V. Scheffer, Partial regularity of solutions to the Navier-Stokes equations, Pacific J. Math. 66 (1976), no. 2, 535-552.

[Ser62] J. Serrin, On the interior regularity of weak solutions of the Navier-Stokes equations, Arch. Rational Mech. Anal. 9 (1962), 187-195.

[SF75] P. L. Sulem and U. Frisch, Bounds on energy flux for finite energy turbulence, J. Fluid Mech. 72 (1975), 417-423.

[SŠ01] G. Seregin and V. Šverák, On solutions to the Navier-Stokes equations with lower bounds on the pressure, Max-Planck Institut fü Mathematik, Leipzig, 2001, preprint no. 92 .

[Str88] M. Struwe, On partial regularity results for the Navier-Stokes equations, Comm. Pure Appl. Math. 41 (1988), no. 4, 437-458.

[Tay91] M. E. Taylor, Pseudodifferential operators and nonlinear PDE, Progress in Mathematics, vol. 100, Birkhäuser Boston Inc., Boston, MA, 1991.

[Tri92] H. Triebel, Theory of function spaces. II, Monographs in Mathematics, vol. 84, Birkhäuser Verlag, Basel, 1992.

[Tsa98] T.-P. Tsai, On Leray's self-similar solutions of the Navier-Stokes equations satisfying local energy estimates, Arch. Rational Mech. Anal. 143 (1998), no. 1, 29-51.

[Wei80] F. B. Weissler, Local existence and nonexistence for semilinear parabolic equations in $L^{p}$, Indiana Univ. Math. J. 29 (1980), no. 1, 79-102.

Department of Mathematics, Penn State University, 218 McAllister Building, UniVERSITY PARK, PA U.S.A. 16802

E-mail address: mazzucat@math.psu.edu 